\title{
Fuzzy Pi and Integrating Type Fuzzy PID Controllers of Linear, Nonlinear and Time -Delay Systems
}

\author{
Prof.K.A.Gopala Rao
}

B.Amarendra Reddy

P.Durga Bhavani

\begin{abstract}
This paper presents the comparison of tracking control performance of fuzzy PI Controller with fuzzy PID and conventional PID controllers. Fuzzy PI controller and Fuzzy PID controller composes of Mamdani type fuzzy controllers and parameters are adjusted to meet the desired control system performances both in transient state and steady state. Computer simulations are done for controlling the time delay, Nonlinear, and Linear systems by using fuzzy PI, fuzzy PID and the conventional PID controller are shown in this paper. The effectiveness of fuzzy controllers over the conventional controller are shown by choosing Gaussian, triangular, pi input fuzzy sets.
\end{abstract}

\section{Index Terms}

FOPDT (First Order plus Time Delay), Fuzzy control Fuzzy PI controller, Fuzzy PID controller, Membership functions.

\section{INTRODUCTION}

Conventional PID controllers have been extensively used in industry, due to their effectiveness for linear systems, ease of design and inexpensive cost. Despite their effectiveness for linear systems, conventional PID controllers are not suitable for nonlinear systems and higher-ordered and time-delayed systems, not to mention complex and vague systems that require expert knowledge. For these reasons, it is worth developing fuzzy-logic-based controllers which have the capability to handle not only linear, as well as illdefined, systems. Mamdani and his coworkers were pioneers in applying fuzzy techniques to process control. The relations between fuzzy and conventional three mode (proportional-integralderivative, PID) controllers have been studied by several authors (Siler and Ying 1989, Ying et al.1990).

Just like the conventional nonfuzzy control which has two-term and three-term control, the conventional fuzzy control also has two-term and three-term control. The fuzzy two-term control has two different types: one is Fuzzy-Proportional-Derivative (FZ-PD) type control which generates control output from error and change in error and is a position type control; another is Fuzzy-ProportionalIntegral (FZ-PI) type control and the design is more difficult. Although some approximations on acceleration

error can reduce the difficulties, the performance is not improved much over FZ-PI because of the small influence of acceleration error in general. Thus, an enhanced control which generates incremental control output from error and change in error and is a velocity type control. The fuzzy three term control is Fuzzy-
Proportional-Integral-Derivative (FZ-PID) type control. The existing one generates incremental control output from error, change in error and acceleration error [3].FZ-PI type control is known to be more practical than FZ-PD type because it is difficult for the FZ-PD to remove steady state error. The FZ-PI type control is, however, known to give poor performance in transient response for higher order process due to the internal integration operation. Theoretically, FZ-PID type control should enhance the performance a lot. However, the existing FZ-PID type control [3] needs three inputs, which will expand the rule-base greatly.

Because of the nonlinear property of control gains, Fuzzy PID type controllers possess the potential to improve and achieve the better system performance over the conventional PID controllers. In fuzzy PID Controllers normally a 3-Dimensional rule base is required. It is difficult to obtain 3-Dimensional information. Fuzzy PID controllers can be constructed in many ways(1) By Using the three input structure,(2) Parallel structure of Fuzzy PI and PD controllers and (3) Integrating the Fuzzy PI and PD controllers in a single rule base structure.

In this paper, fuzzy PID controller has been employed to meet the desired performances by tuning the controller parameters appropriately. The Mamdani fuzzy PI controller consists of two inputs, error and change of error. The Fuzzy PID controller is used to control the time delay, Nonlinear and Linear Systems and their simulation results are shown by comparing with the fuzzy PI and conventional PID controllers.

This paper is organized as follows; section II describes the principal components of fuzzy PI and Fuzzy PID controller, section III describes fuzzification algorithm, fuzzy control rules and defuzzification algorithm, section IV describes the computer simulations.

\section{II.FUZZY PI AND PID CONTROL SYSTEM STRUCTURE}

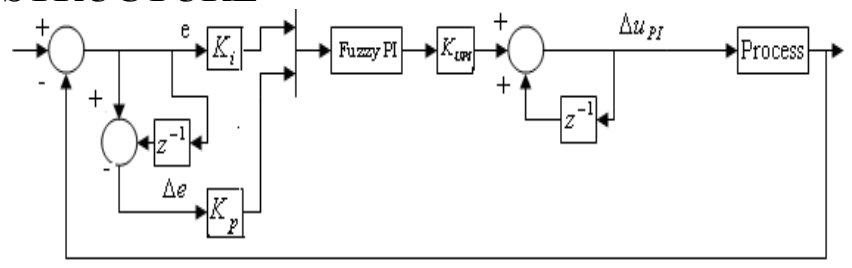

Fig.1. Structure of FZ-PI control 


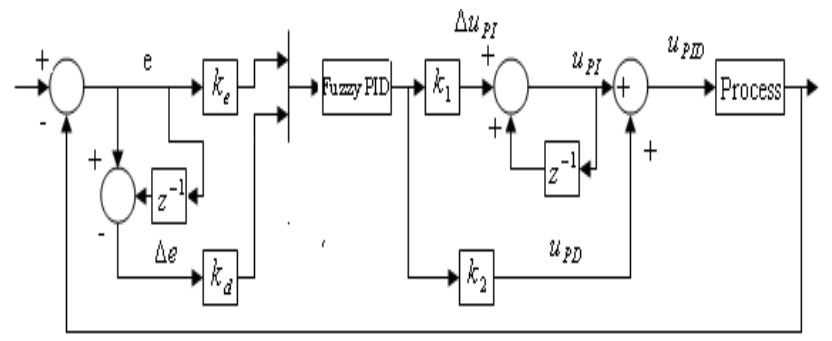

Fig.2. Structure of Fuzzy PID control

The structure of Fuzzy PI and Fuzzy PID controllers are shown in

Fig.1 and Fig.2 respectively.

\section{A. Derivation of the fuzzy PI control law}

The output of the conventional analog PI controller in the frequency s- domain, is given by

$$
u_{P I}(s)=\left(K_{p}^{c}+\frac{K_{i}^{c}}{s}\right) E(s),
$$

Where $K_{p}^{c}$ and $K_{i}^{c}$ are the proportional and integral gains, respectively, and $\mathrm{E}(\mathrm{s})$ is the tracking error signal. This equation can be transformed into the discrete version by applying the bilinear transformation $s=(2 / T)(z-1 / z+1)$, where $T>0$, is the sampling period, which results in the following form:

$$
\Delta u_{P I}(n T)=K_{p} e_{v}(n T)+K_{i} e_{p}(n T),
$$

Where

$$
\begin{aligned}
& \Delta u_{P I}(n T)=\frac{u_{P I}(n T)-u_{P I}(n T-T)}{T}, \\
& e_{v}(n T)=\frac{e(n T)-e(n T-T)}{T},
\end{aligned}
$$

And

$$
e_{p}(n T)=e(n T)
$$

More precisely, $\Delta u_{P I}(n T)$ is the incremental control output of the PI controller, $e_{v}(n T)$ is the rate of change of error signal, and $e_{p}(n T)$ is the error signal.

We can rewrite (2) as

$$
u_{P I}(n T)=u_{P I}(n T-T)+T \Delta u_{P I}(n T),
$$

In the design of the fuzzy PI controller to be discussed later, we replace the term $T \Delta u_{P I}(n T)$ with $k_{U P I} \Delta u_{P I}(n T)$.

$$
u_{P I}(n T)=u_{P I}(n T-T)+K_{U P I} \Delta u_{P I}(n T),
$$

Where $K_{U P I}$ is a fuzzy control gain.

To avoid having to use the $\Delta^{2} e$ input, a hybrid velocity/position type PID algorithm is presented as in eq.(5).

$$
u_{k}^{P I D}=u_{k}^{P I}+u_{k}^{P D}
$$

Where $u_{k}^{P I}$ is the velocity type PI control and $u_{k}^{P D}$ is the position type PD control.

$$
u_{k}^{P I}=u_{k-1}^{P I}+\Delta u_{k}^{P I}
$$

$$
\begin{gathered}
\Delta u_{k}^{P I}=k_{1} e_{k}+k_{p} \Delta e_{k} \\
u_{k}^{P D}=k_{p} e_{k}+k_{D} \Delta e_{k}
\end{gathered}
$$

The gains of the fuzzy three-term control can be expressed as shown in eq.(8).

$$
\begin{gathered}
k_{y}=k_{1} F\left\{k_{d}\right\}+k_{2} F\left\{k_{e}\right\} \\
k_{I}=k_{1} F\left\{k_{e}\right\} \\
k_{D}=k_{2} F\left\{k_{d}\right\}
\end{gathered}
$$

$\mathrm{F}\{\}$ represents the fuzzy operation.

\section{FUZZIFICATION, CONTROL RULE BASE AND \\ DEFUZZIFICATION}

\section{A. Fuzzification}

In the fuzzification stage of fuzzy PI controller, e(nT) and $\Delta \mathrm{e}(\mathrm{nT})$ are the controller inputs. The number of input membership functions used in input scaling are three (n: negative, p:positive and z:zero). The membership functions of two inputs are similarly selected as shown in fig. 3 . Where $\mathrm{L}$ is the adjustable constant

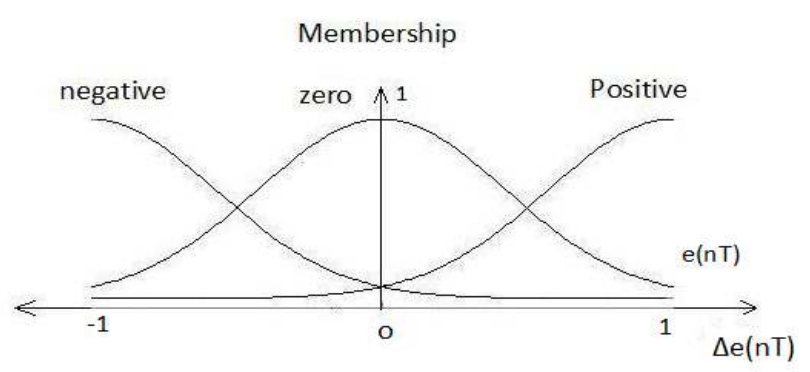

Fig.3 Input membership function for Fuzzy PI and PID controllers 


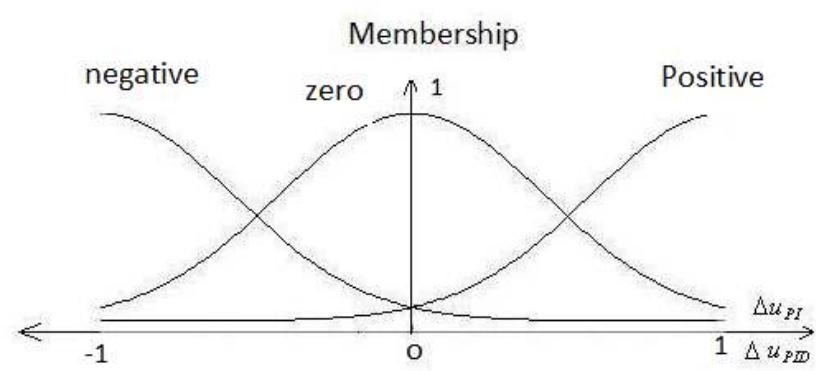

Fig.4 Output membership function for Fuzzy PI and PID controllers

\section{B. Fuzzy Rules}

The output membership functions for $\Delta u_{P I}$ and $\Delta u_{P I D}$ are selected as shown in Fig.4.The number of output membership functions is three ( $h_{n}$ : negative, $h_{z}$ : zero, $h_{p}$ : positive). Based on the input membership functions in fuzzification stage and these output membership functions, the number of fuzzy control rules for the Mamdani fuzzy PI controller are nine, which are

$\mathrm{R} 1:$ IF $e(n T)$ is $\mathrm{p}$ AND $\Delta e(n T)$ is $\mathrm{p}$ THEN

$$
\Delta u_{P I} \text { is } h_{p}
$$

R2: IF $e(n T)$ is z AND $\Delta e(n T)$ is $\mathrm{p}$ THEN

$$
\Delta u_{P I} \text { is } h_{p}
$$

R3: IF $e(n T)$ is n AND $\Delta e(n T)$ is p THEN

$$
\Delta u_{P I} \text { is } h_{z}
$$

R4: IF $e(n T)$ is n AND $\Delta e(n T)$ is z THEN

$$
\Delta u_{P I} \text { is } h_{n}
$$

R5: IF $e(n T)$ is z AND $\Delta e(n T)$ is z THEN

$$
\Delta u_{P I} \text { is } h_{z}
$$

R6: IF $e(n T)$ is $\mathrm{p}$ AND $\Delta e(n T)$ is z THEN

$$
\Delta u_{P I} \text { is } h_{p}
$$

R7: IF $e(n T)$ is n AND $\Delta e(n T)$ is n THEN

$$
\Delta u_{P I} \text { is } h_{n}
$$

R8: IF $e(n T)$ is z AND $\Delta e(n T)$ is n THEN

$$
\Delta u_{P I} \text { is } h_{n}
$$

R9: IF $e(n T)$ is $\mathrm{p}$ AND $\Delta e(n T)$ is n THEN

$$
\Delta u_{P I} \text { is } h_{z}
$$

Here AND is Zadeh's logical “AND” defined by

$$
\mu_{A} \operatorname{AND} \mu_{B}=\min \left\{\mu_{A}, \mu_{B}\right\}
$$

Where $\mu_{A}$ and $\mu_{B}$ are the membership functions of fuzzy sets A and $\mathrm{B}$ respectively.
To reduce the complexity of the rule-base design and gain tuning, a common rule-base for both FZ-PI and FZ-PD parts is used in this paper. The rule-base design for FZ-PID control is the same as for FZ-PI control.

\section{Defuzzification}

To convert the fuzzy sets to the real numbers, the centroid defuzzifier is used to calculate the output change $\Delta u_{P I}(\mathrm{nT})$ of the Fuzzy PI controller.

$$
\Delta u_{P I}(n T)=\frac{\sum \mathrm{h}_{\mathrm{i}} \mu_{\mathrm{i}}}{\sum \mu_{\mathrm{i}}}
$$

where $h_{i}$ is the value of the output member for $i^{\text {th }}$ rule, $\mu_{i}$ is the output membership value for $i^{\text {th }}$ rule.

\section{ANALYTICAL INPUT-OUTPUT RELATIONSHIP}

Due to the use of Zadeh AND Operator, the input space must be divided into a number of regions in such a way that in each region a unique relationship can be obtained for each fuzzy rule between the two membership functions being ANDed. First consider the rule antecedent parts which contain two membership functions the boundary on which the membership value is same between two membership functions is obtained by letting them equal. Once the boundary is available it is trivial to determine which function belongs to which IC compute the value of $e(n T)$ and $\Delta e(n T)$ using one pair of error and rate in either IC.

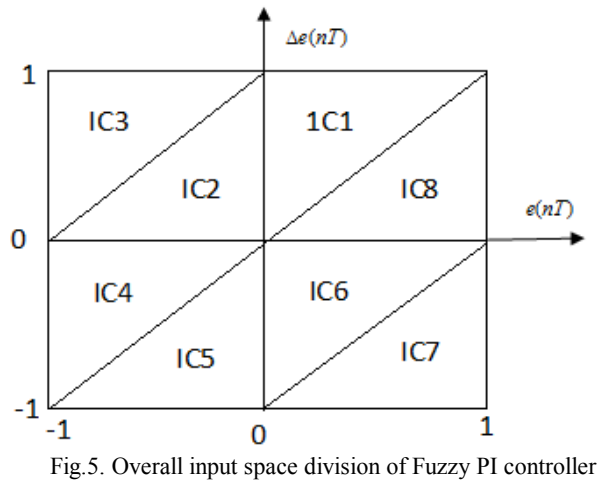

Superimposing all the individual input space divisions to form overall input space division for all rules in fuzzy PI controller is shown in fig.5. The control rules for the fuzzy PI controller (R1)(R9), with membership functions and input-combination (IC) regions together, are used to evaluate appropriate fuzzy control laws for each region. Working through all regions, we obtain the following formulas for the four IC regions and are shown in below. 
IC $1=\frac{2 e^{-0.5 p_{3}} \cdot h_{p}+e^{-0.5 q_{3}} \cdot h_{p}+2 e^{-0.5 p_{1}} \cdot h_{n}+e^{-0.5 q_{2}} \cdot h_{z}+2 e^{-0.5 p_{2}} \cdot h_{z}+e^{-0.5 q_{1}} \cdot h_{n} \text { membership functions with conventional PID controller. These }}{2 e^{-0.5 p_{3}}+e^{-0.5 q_{3}}+2 e^{-0.5 p_{1}}+e^{-0.5 q_{2}}+2 e^{-0.5 p_{2}}+e^{-0.5 q_{1}}}$ responses are shown in Fig.6.-Fig.8.

$I C 2=\frac{3 e^{-0.5 p_{3}} \cdot h_{p}+2 e^{-0.5 q_{3}} \cdot h_{p}+e^{-0.5 q_{2}} \cdot h_{z}+2 e^{-0.5 p_{2}} \cdot h_{z}+e^{-0.5 p_{1}} \cdot h_{n}}{3 e^{-0.5 p_{3}}+2 e^{-0.5 q_{3}}+e^{-0.5 q_{2}}+2 e^{-0.5 p_{2}}+e^{-0.5 p_{1}}}$

$I C 3=\frac{3 e^{-0.5 p_{3}} \cdot h_{p}+e^{-0.5 q_{3}} \cdot h_{p}+2 e^{-0.5 p_{1}} \cdot h_{n}+3 e^{-0.5 p_{2}} \cdot h_{z}}{3 e^{-0.5 p_{3}}+e^{-0.5 q_{3}}+2 e^{-0.5 p_{1}}+3 e^{-0.5 p_{2}}}$

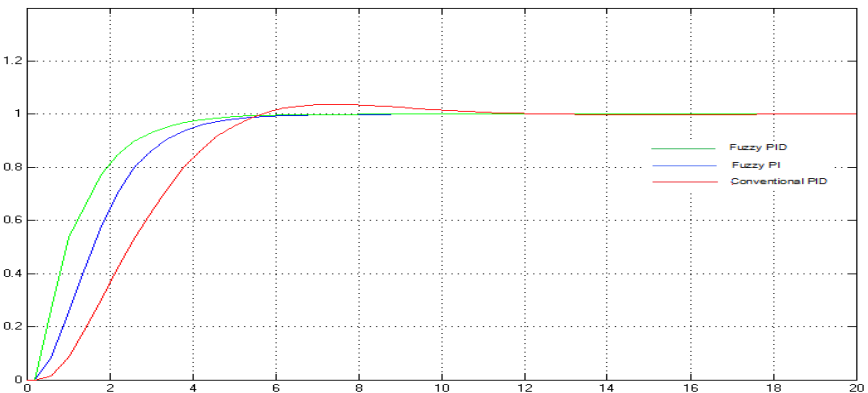

Fig.6. Unit step response for "Gaussian" membership function

$I C 4=\frac{3 e^{-0.5 p_{3}} \cdot h_{p}+2 e^{-0.5 q_{3}} \cdot h_{p}+e^{-0.5 p_{1}} \cdot h_{n}+e^{-0.5 q_{2}} \cdot h_{z}+2 e^{-0.5 p_{2}} \cdot h_{z}}{3 e^{-0.5 p_{3}}+2 e^{-0.5 q_{3}}+e^{-0.5 p_{1}}+e^{-0.5 q_{2}}+2 e^{-0.5 p_{2}}}$

$I C 5=\frac{3 e^{-0.5 q_{3}} \cdot h_{p}+2 e^{-0.5 q_{2}} \cdot h_{z}+2 e^{-0.5 p_{3}} \cdot h_{p}+e^{-0.5 q_{1}} \cdot h_{n}+e^{-0.5 p_{2}} \cdot h_{z}}{3 e^{-0.5 q_{3}}+2 e^{-0.5 q_{2}}+2 e^{-0.5 p_{3}}+e^{-0.5 q_{1}}+e^{-0.5 p_{2}}}$

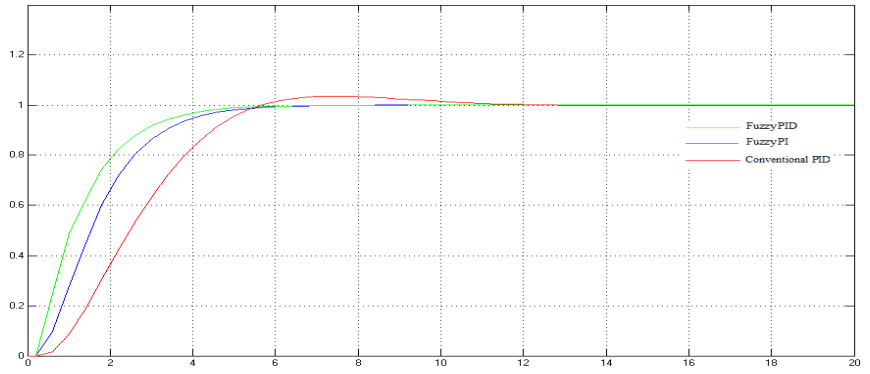

IC6 $=\frac{e^{-0.5 p_{3}} \cdot h_{p}+2 e^{-0.5 q_{3}} \cdot h_{p}+e^{-0.5 p_{1}} \cdot h_{n}+2 e^{-0.5 q_{2}} \cdot h_{z}+e^{-0.5 p_{2}} \cdot h_{z}+2 e^{-0.5 q_{1}} \cdot h_{n}}{e^{-0.5 p_{3}}+2 e^{-0.5 q_{3}}+e^{-0.5 p_{1}}+2 e^{-0.5 q_{2}}+e^{-0.5 p_{2}}+2 e^{-0.5 q_{1}}}$

Fig.7. Unit step response for "Triangular" membership function

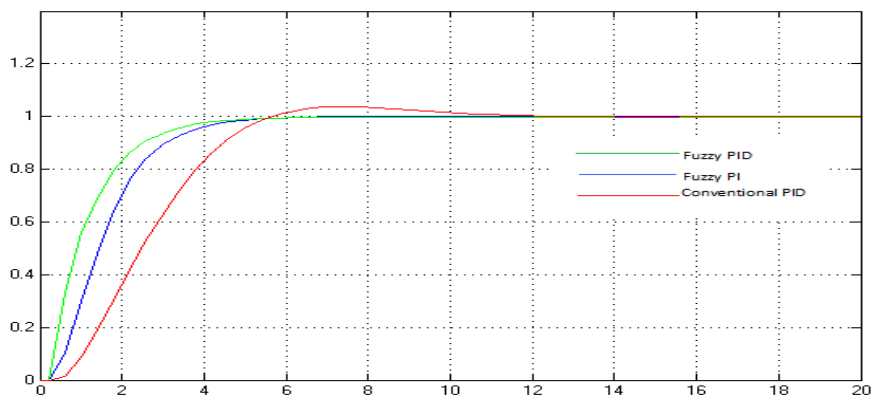

Fig.8. Unit step response for "Pi" membership function

Where $p_{1}=\left(\frac{e(n)-1}{0.426}\right)^{2}, p_{2}=\left(\frac{e(n)}{0.426}\right)^{2}, p_{3}=\left(\frac{e(n)+1}{0.426}\right)^{2}$

$$
q_{1}=\left(\frac{r(n)-1}{0.426}\right)^{2}, q_{2}=\left(\frac{r(n)}{0.426}\right)^{2}, q_{3}=\left(\frac{r(n)+1}{0.426}\right)^{2}
$$

Consider a first order linear process described by $G(s)=\frac{1}{s+1}$. The control performance of the system subjected to a unit step input is compared by considering Fuzzy PID, Fuzzy PI controllers using gaussian, triangular and pi membership functions with conventional PID controller. These responses are shown in Fig.9-Fig.11.

\section{V.SIMULATION RESULTS}

Consider as a first example a First Order Time Delay Process described by $\mathrm{G}(\mathrm{s})=\frac{e^{-0.2 s}}{s+1}$. The control performance of the system subjected to a unit step input is compared by considering Fuzzy PID, Fuzzy PI controllers using Gaussian, triangular and pi 


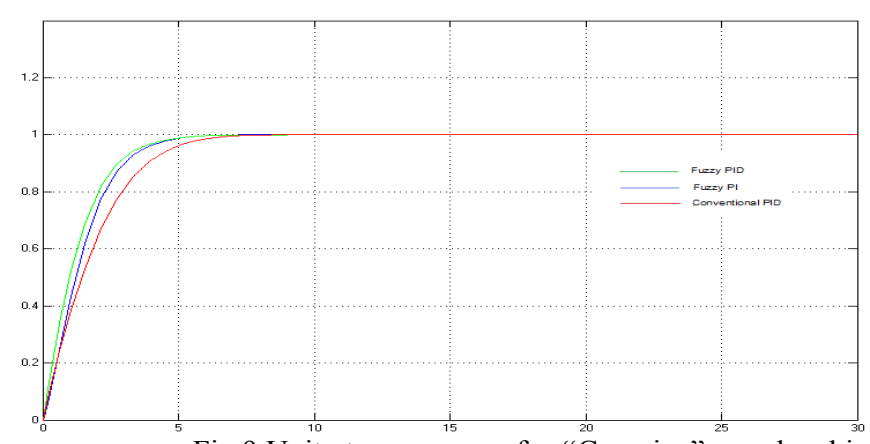

Fig.9.Unit step response for "Gaussian" membership

function

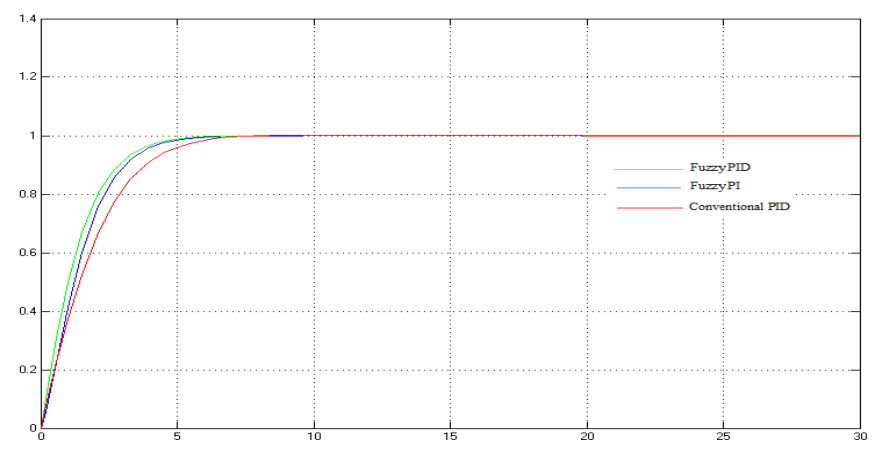

Fig.10. Unit step response for "Triangular" membership

function

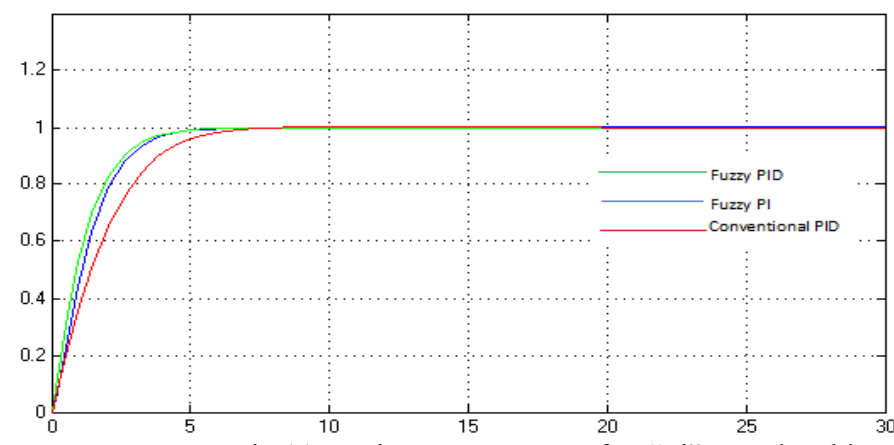

Fig.11. Unit step response for "Pi" membership

function

Consider a second order linear process described by $G(s)=\frac{1}{s^{2}+2 s+1}$. The control performance of the system subjected to a unit step input is compared by considering Fuzzy PID, Fuzzy PI controllers using Gaussian, triangular and pi membership functions with conventional PID controller. These responses are shown in Fig.12-Fig.14.

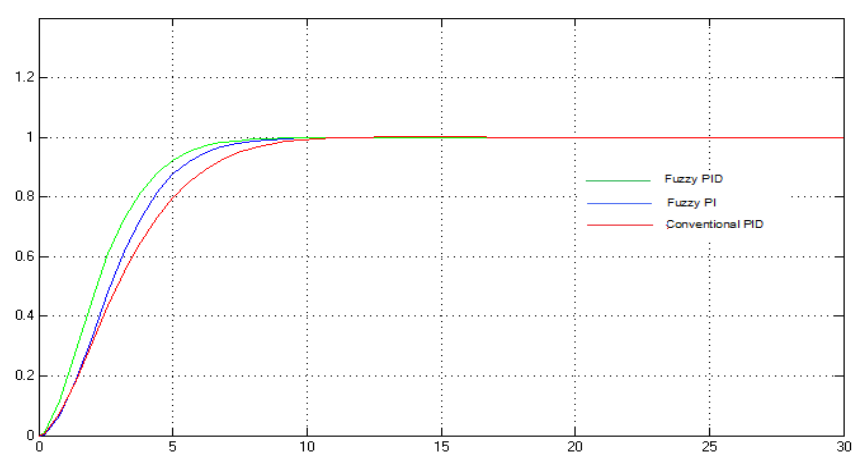

Fig.12. Unit step response for "Gaussian" membership function

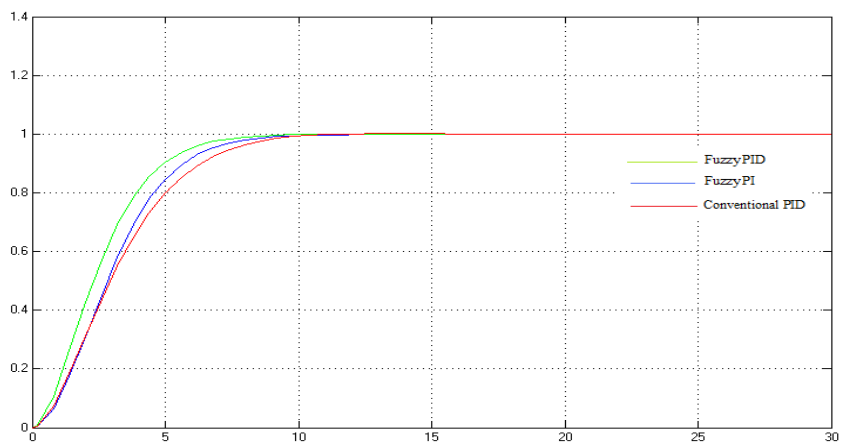

Fig.13. Unit step response for "Triangular" membership function

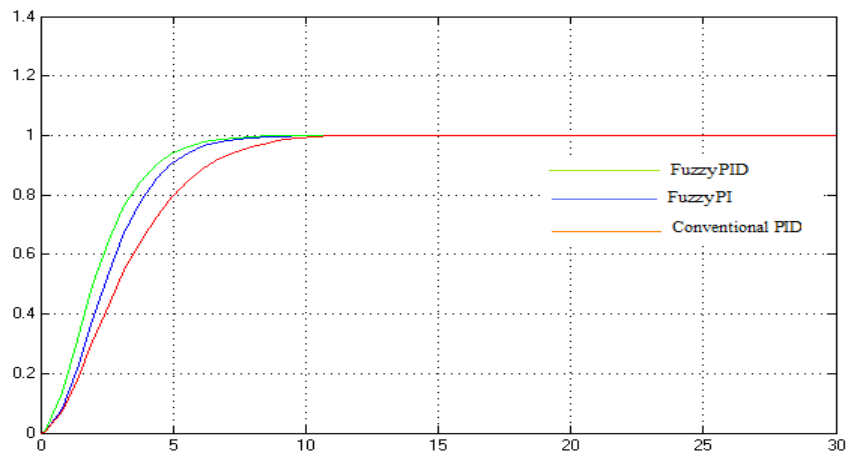

Fig.14. Unit step response for "Pi" membership function

Consider a third order linear process described by $G(s)=\frac{10}{0.04 s^{3}+0.54 s^{2}+1.5 s+1}$. The control performance of the system subjected to a unit step input is compared by considering Fuzzy PID, Fuzzy PI controllers using Gaussian, triangular and pi membership functions with conventional PID controller. These responses are shown in Fig.15Fig. 17. 


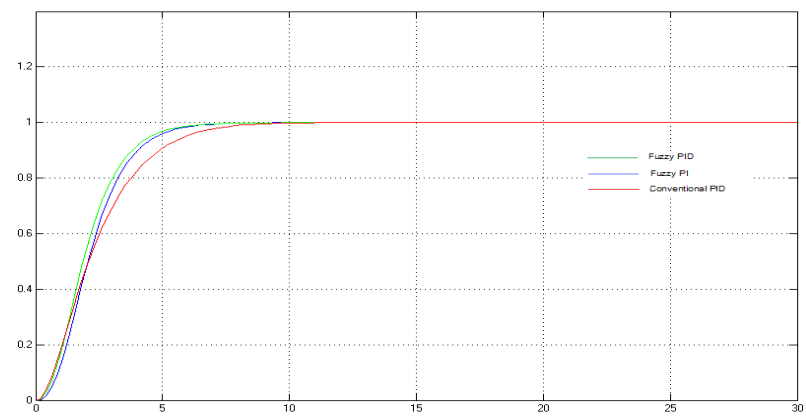

Fig.15. Unit step response for "Gaussian" membership function

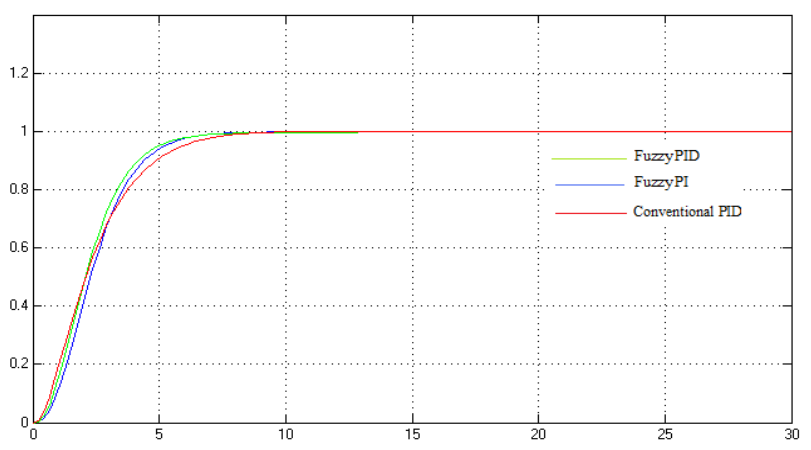

Fig.16. Unit step response for "Triangular" membership function

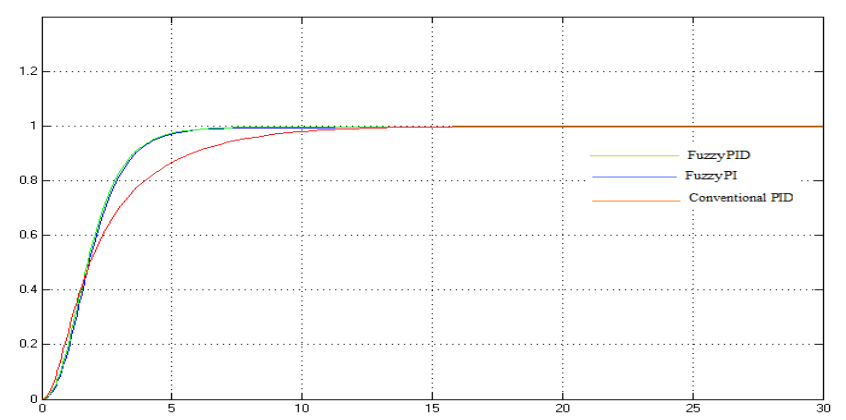

Fig.17. Unit step response for "Pi" membership function

Consider a nonlinear system described by

$\dot{y}=-\mathrm{y}(\mathrm{t})+\sqrt{|y(t)|}+\mathrm{u}(\mathrm{t})$. The control performance of the system subjected to a unit step input is compared by considering Fuzzy PID, Fuzzy PI controllers using Gaussian, triangular and pi membership functions with conventional PID controller. These responses are shown in Fig.18-Fig.20.

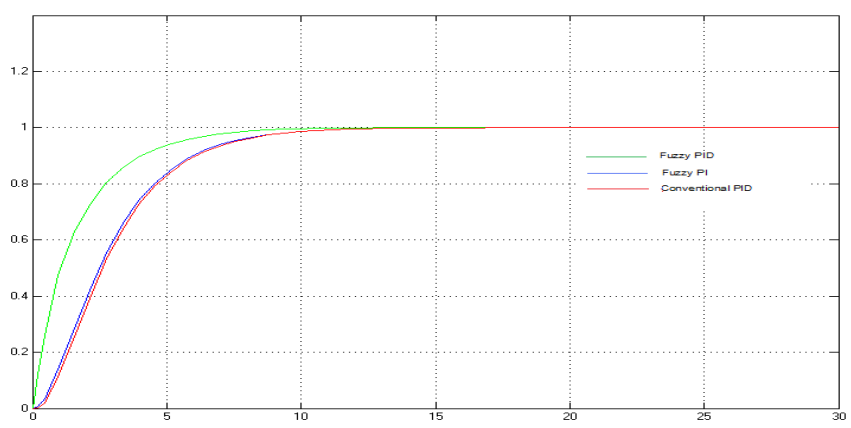

Fig.18. Unit step response for "Gaussian" membership function

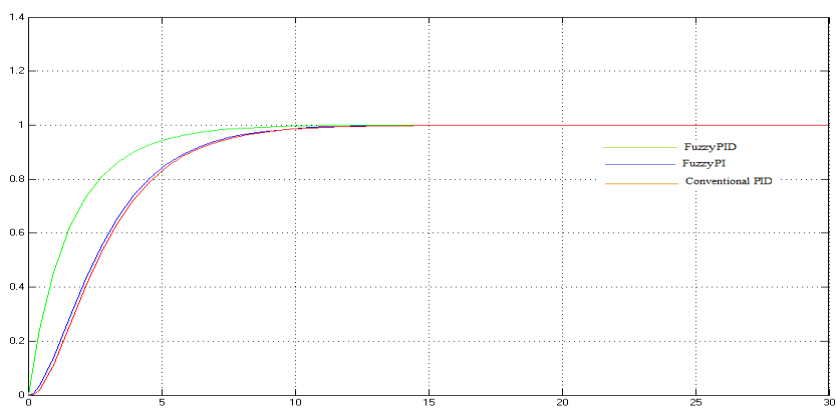

Fig.19. Unit step response for "Triangular" membership function

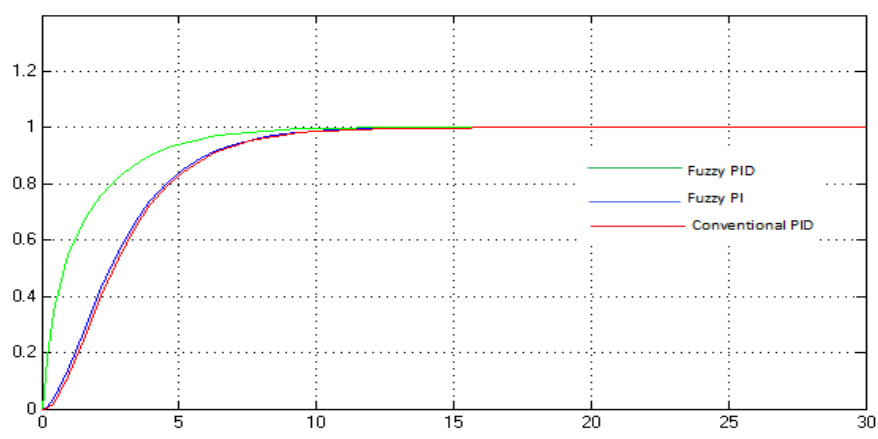

Fig.20. Unit step response for "Pi" membership function

\section{CASE STUDY:}

A P.M.D.C Motor controlled servo mechanism is considered to demonstrate the efficacies of fuzzy controllers[7]. The open loop transfer function is defined as

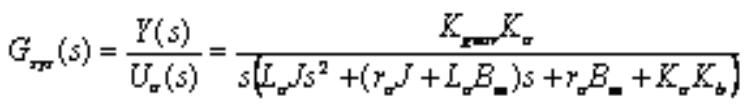

The parameters [7] used for simulations are as follows:

$$
\text { Let } r_{a}=5, L_{a}=0.01, K_{a}=0.2, \mathrm{j}=0.0005 \text {, }
$$

$$
B_{m}=0.00001, K_{\text {gear }}=0.05, K_{b}=0.2 \text {. }
$$

The control performance of the system subjected to a unit step input is compared by considering Fuzzy PID, Fuzzy PI controllers using Gaussian, triangular and pi membership functions 
with conventional PID controller. These responses are shown in Fig.21-Fig.23.

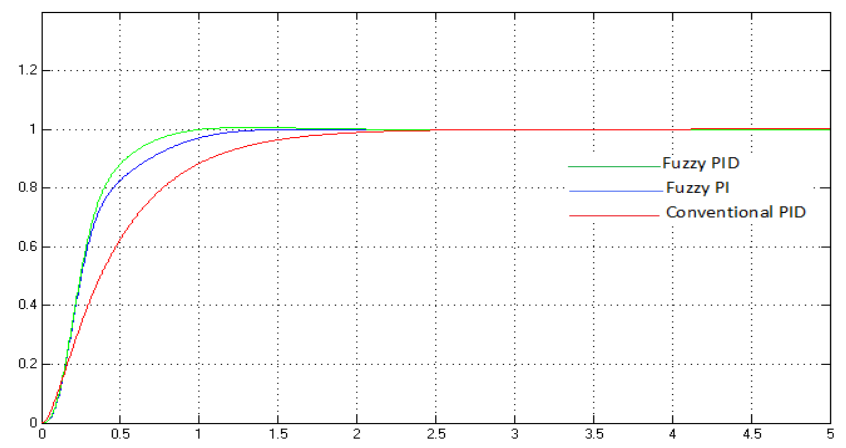

Fig.21. Unit step Response for "Triangular" membership function

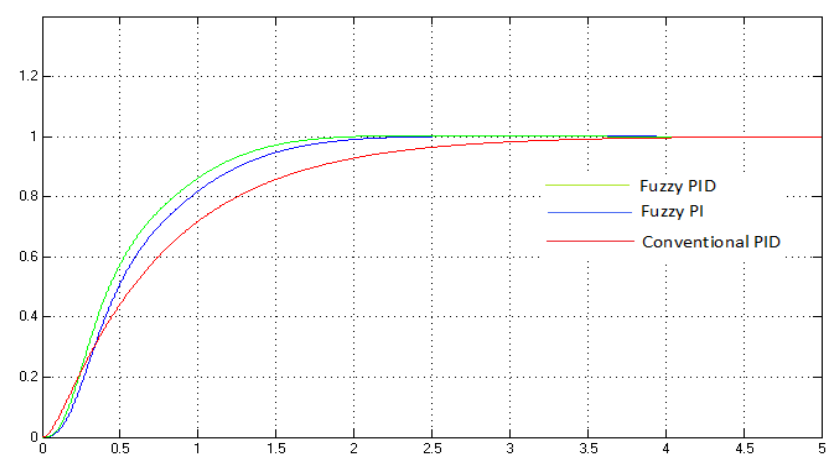

Fig.22. Unit step Response for "Gaussian" membership function

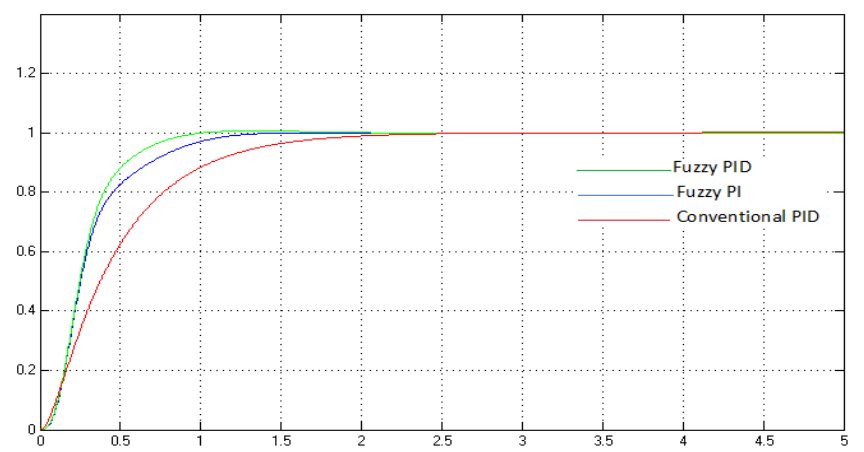

Fig.23. Unit step .Representation of "Pi" membership function

\section{CONCLUSIONS}

Simulation results are shown for the control performances of Fuzzy PI controller, Fuzzy PID Controller and Conventional PID Controller by considering time delay, nonlinear and linear systems and a PMDC Servo mechanism In both fuzzy PI and fuzzy PID controllers, 'Gaussian', 'triangular' and 'pi' membership functions are considered and the step responses are observed. Irrespective of the different types of systems and the different membership functions the Fuzzy PID Controller is showing better performance than Fuzzy PI Controller. It is also observed that Fuzzy Controllers are showing better performance than Conventional PID Controller.

\section{REFERENCES}

1. E.Cox, "Fuzzy Fundamentals", IEEE spectrum,pp.5861,10/1992.

2. C.C.Lee, "Fuzzy Logic in control systems:Fuzzy Logic Controller",IEEE transactions on systems, man and cybernetics,vol.20,no.2,pp.404-434,03/1990.

3. G.M.Abdelnour,el at. "Design of a Fuzzy Controller Using Input and Output Mapping Factors",IEEE transactions on systems, man and cybernetics, vol.21, no.5, 09/1991, pp.952-960.

4. J.Lee, "On Methods for Improving Performance of PIType Fuzzy Logic Controller”,IEEE transactions on fuzzy systems,vol.1, no.4, 11/1993, pp.298-301.

5. H.X.Li,H.B.Gatland, "A New Methodology for Designing a fuzzy Logic Controllers”, IEEE transactions on fuzzy sytems, manand cybernetics,vol.25, no.2, 02/1995.

6. K.J.A.strom, T.Hagglund,"Automatic Tuning of PID Controllers",Instrument society of America, 1988,pp.7-8.

7. H.X. Li, H.B. Gatland, enhancement methods of fuzzy logic control, in: Proceedings of the Fuzz-IEEE/IFES'95, Vol.1, Yokohama, Japan, pp. 331-336, 1995. 